\title{
Complete Congenital Heart Block in a Newborn Associated with Maternal Systemic Lupus Erythematosus: A Case Report
}

\author{
Mahmoud A. Kiblawi,' Alina Naeem,' Elham A. Al Attrash,' Subhranshu Kar,' B. K. M. Goud'
}

\begin{abstract}
Background: Complete Congenital Heart Block (CCHB) is a rare disease of the newborn that carries significant morbidity and mortality. CCHB can be diagnosed early or late in life. In newborns, it is usually associated with maternal autoantibodies or a congenital cardiac structural abnormality. The most common presentation of CCHB is bradycardia that can be diagnosed by an electrocardiogram. Results: This is a case report of a male infant born to a mother with an autoimmune disease, Systemic Lupus Erythematosus (SLE), who was found to have third degree heart block at birth. Conclusion: Early diagnosis and prompt management of the case is important for a better prognosis and prevention of associated complications. Neonates with CCHB should be managed at a tertiary care center and the only definite treatment is insertion of a pacemaker. Moreover, prenatal diagnosis and specific obstetric counseling of pregnant women with SLE along with careful monitoring with serial ultrasonography and echocardiography are of paramount importance in prevention of the disease in subsequent offspring.
\end{abstract}

Keywords: Congenital Heart Block, Systemic Lupus Erythematosus, Bradycardia, SS-A Antibodies (Source: MeSH, NLM)

\section{Introduction}

Complete Congenital heart block (CCHB) is a rare clinical entity present in about 1 in 20,000 live births and has significant morbidity and mortality.' It generally occurs due to the presence of maternal auto-antibodies, anti-Ro/SSA and anti-La/SSB, that are transferred across the placenta to the fetus and affect the fetal heart. More rarely, it may be associated with a congenital structural abnormality of the heart. ${ }^{2-3}$ The association between CCHB and maternal autoantibodies has been documented in different case reports..$^{4-8}$

About the Author: Mahmoud Ahmed Kiblawi, recently graduated from a MBBS program at RAK Medical and Health Sciences University, Ras Al Khaimah, UAE, where he was honored thrice for best academic performance.
The risk of Neonatal Lupus Erythematosus (NLE) or Congenital Heart Block (CHB) is $1 \%$ in infants with positive maternal autoantibodies to SSA/Ro. The risk is much higher if the mother already has had a child with NLE or has hypothyroidism due to thyroid autoantibodies that test positive for anti-Ro/SSA antibodies. ${ }^{2}$ The most common clinical manifestations of NLE are related to the cardiac, dermatologic and hepatobiliary systems. Some infants may also have hematologic, central nervous system or splenic abnormalities. ${ }^{9-10}$ Infants with NLE have $15-30 \%$ risk of developing CHB. ${ }^{2,1}$

In the early part of 19th century, congenital heart block was clinically recognized by slow pulse, syncopal attacks and ECG findings consistent with complete atrio-ventricular block leading to the death of the child early on in infancy. ${ }^{12-13}$ Considerable information has also been accumulated since then concerning the clinical profile and circulatory hemodynamics in such cases. ${ }^{14-15}$ Mortality is highest in the neonatal period and association with major cardiac malformations is a bad prognos- tic sign. ${ }^{16}$ We present a rare case of Complete Congenital Heart Block (CCHB) in a newborn associated with maternal Systemic Lupus Erythematosus (SLE). A written informed consent was obtained from the parents of the patient for the publication of the available data.

\section{The Case}

A 3-days-old full-term male infant, born out of a consanguineous marriage by normal vaginal delivery with a birth weight of $3,330 \mathrm{~g}$, presented with bradycardia (heart rate of 40-65 beats/min) and respiratory distress for which he was admitted to special care baby unit (SCBU). His mother, Gravida 6 and Para 6, had a past medical history significant for Systemic Lupus Erythematosus (SLE) as positive anti-Ro/SS-A antibodies had been discovered 5 years ago. Her antibody titers have been persistently elevated since then. Furthermore, she has had persistent hypothyroidism over the past four years which currently being treated with levothyroxine. Also, two of her previously offspring were found positive when tested for Antinuclear Antibody (ANA) and anti-Ro/SSA antibodies.

The infant was stabilized in the SCBU and kept on two liters of oxygen through nasal cannula for respiratory distress. He was tolerating feeds. His heart rate ranged between 40-65 beats/ min despite being given two doses of atropine ( $0.1 \mathrm{mg} /$ dose). Laboratory examinations showed capillary blood sugar of 72 $\mathrm{mg} / \mathrm{dl}$, normal complete blood count and normal electrolyte levels (Na- $137 \mathrm{meq} / \mathrm{L}, \mathrm{K}-3.6 \mathrm{meq} / \mathrm{L})$. At presentation, the arterial blood gas analysis $\left(\mathrm{pH}^{-7.25}, \mathrm{pCO}_{2}-58 \mathrm{mmHg}, \mathrm{pO}_{2}-40 \mathrm{mmHg}\right.$, $\mathrm{HCO}_{3}-24 \mathrm{mmHg}$ and base excess of -4 ) showed respiratory aci- 


\section{Case Report}

Figure 1. Anteroposterior (AP) Chest Radiograph of the Infant

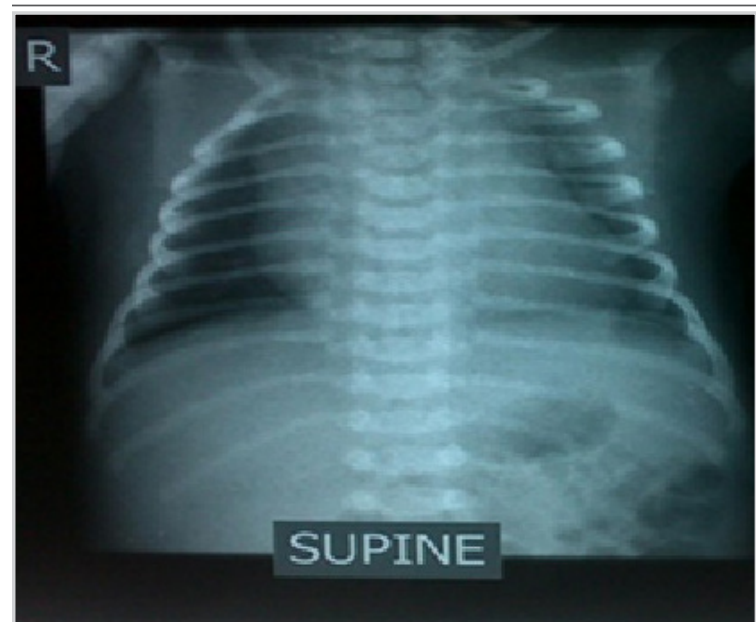

Note: Chest radiograph showed cardiac enlargement.

Figure 2. ECG from Infant Demonstrating Complete Congenital Heart Block

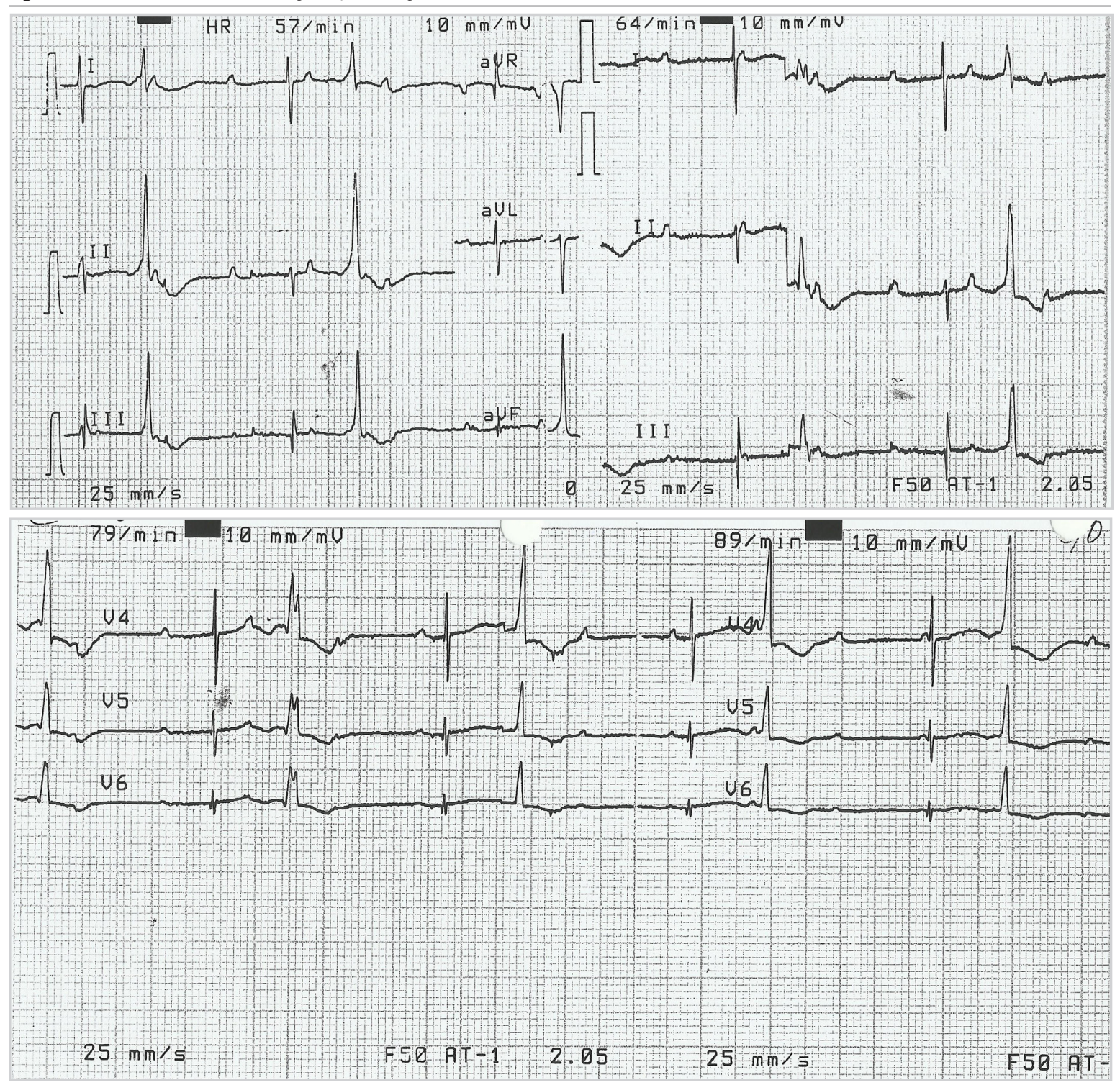

Note: ECG of the infant demonstrates no relation between P wave and QRS complex. The P wave has the normal rate and is regular, as it is arising from the sino-atrial node. The QRS complex illustrates the ventricular escape rhythm and hence is very slow. dosis. Chest X-ray AP view showed cardiac enlargement (Figure heart block (Figure 2). Based on his condition, it was decided to do further evaluation and management (including the possibility of pacemaker insertion) to support the patient in the best possible way. Echocardiography was performed and it revealed no structural abnormality. A permanent pacemaker (St. Jude VVI PM at rate 90 beats/min) implantation was done on the 8 th day of the infant's life. Upon further follow up, the patient was stable and his general health status was good. The pacemaker was functioning well at a rate of 90 beats/min. He was thriving well with normal development.

\section{Discussion}

SLE is an autoimmune disease characterized by production of large amounts of circulating autoantibodies that can affect any organ of the body and leads to variable clinical manifestations. The ANA is the most sensitive laboratory test for the diagnosis of SLE. ${ }^{17}$ The presence of anti-double stranded DNA antibodies 1). His electrocardiogram (ECG) showed features of complete 
and anti- Smith antibodies are more specific for the diagnosis of SLE as they have high sensitivity. Other nonspecific subtypes of ANA can be associated with SLE, including antibodies to Ro (SSA) and La (SSB), but these are also seen in patients with Sjogren's syndrome. Studies have shown that congenital heart block is associated with maternal connective tissue diseases such as SLE and in patients with structural heart disease such as L-transposition of the great arteries. ${ }^{18-19} \mathrm{CCHB}$ can also be observed in patients with neonatal lupus syndrome diagnosed in utero. ${ }^{20}$

Among the conduction defects resulting from maternal SLE, congenital heart block in infants born to mothers with anti-Ro antibodies is the most commonly seen in clinical practice. ${ }^{21}$ The anti Ro antibodies have been detected in maternal sera in up to $98 \%$ of cases of CCHB. ${ }^{22}$ Antibodies to Ro/SSA are detected by enzyme-linked immunosorbent assay (ELISA) and Western blot immunodiffusion tests. The Ro/SSA autoantigen consists of two proteins, R052 and R0660, with the former having close correlation with the development of CCHB. ${ }^{23}$ These antibodies induce myocarditis which leads to CCHB by destroying the cardiac conduction system at the atrioventricular node and bundle of His region. Atrioventricular (AV) block occurs due to impairment in the conduction system causing delay or block in transmission of atrial impulse to ventricles. Third-degree heart block, whether its congenital or acquired, is diagnosed when there is no association between $\mathrm{P}$ wave and QRS complexes. In less than $10 \%$ of cases, congenital AV block may be either 1st or 2nd degree block at birth, of which $50 \%$ progress to 3rd-degree block in postnatal life. ${ }^{15}$ Congenital heart block can be detected in utero between 18 and 28 weeks of gestation by echocardiography in the presence of fetal bradycardia. ${ }^{24-25}$ It may also be associated with other cardiac abnormalities such as endocardial fibroelastosis and dilated cardiomyopathy. ${ }^{26}$ As the fetal heart rate is an important indicator for the prognosis during the newborn period, the persistent bradycardia may eventually lead to increased size of both right and left ventricles. ${ }^{16}$ In children under three years of age, a heart rate less than 100 beats $/ \mathrm{min}$ is considered as bradycardia.' Our patient had a heart rate of 40-65 beats/min, whereas normal newborns have a heart rate of 120-160 beats/min. In spite of his low heart rate, his birth weight was within normal limits. The ECG showed the most severe type of heart block, third degree heart block, where there is no transmission of atrial impulse to the ventricle. CCHB is the most serious complication associated with maternal SLE and once established it is irreversible. ${ }^{3}$

Occasionally, these cases are symptomatic in the neonatal period, as evident in our case, and they are considered high-risk patients requiring urgent intervention. The mortality is highest during the neonatal period. Clucocorticoid therapy is suggested for management of CCHB in utero and in the perinatal period (if associated with anti-Ro and anti-La antibodies), but the therapy itself carries high risk to both the mother and the fetus. ${ }^{27}$ Two third of all congenital heart block patients require pacemaker implantation and it is recommended in symptomatic bradycardia. Pacemaker therapy is recommended in infants with CCHB and heart rates less than 55 beats/min in the absence of structural heart disease or with rates less than 70 beats $/ \mathrm{min}$ with concomitant congenital heart disease or signs of ventricular dysfunction. Beyond the first year of life, a heart rate less than 50 beats/min or abrupt pauses in ventricular rate merit consideration of pacing therapy. Evidence of cardiomyopathy with ventricular dysfunction, complex ventricular ectopy and wide complex escape rhythm are also indications for pacing. ${ }^{28}$ The primary goal of the pacemaker is to prevent sudden cardiac death and to provide symptomatic relief with improvement in quality of life and functional capacity, as was done in our case. ${ }^{22,29}$

\section{Conclusion}

In conclusion, anticipation of CCHB in cases of maternal SLE is important. Early detection of CCHB by health care professionals and timely management is the key to the success of the infant's survival. A thorough physical examination and an ECC can be diagnostic in patients presenting with bradycardia. For prevention, it is necessary to implement: 1) Health education and creating awareness among high risk populations, 2) Emphasize good antenatal care and proper screening, especially in underprivileged populations, because frequent assessment of fetal heart rate by echocardiography during pregnancy is beneficial for a patient with positive anti-Ro and anti-La antibodies, and 3) implementing proper obstetric counseling and support to the family. 


\section{References}

1. Michaëlsson M, Engle MA. Congenital complete heart block: An international study of the natural history. Cardiovasc Clin. 1972;4(3):85-101.

2. Hon KL, Leung AK. Neonatal lupus Erythematosus. Autoimmune Dis. 2012;2012:301274.

3. Admani S, Krakowski AC. Neonatal Lupus Erythematosus Presenting as Atypical Targetoid-like Lesions Involving Cenitals and Soles of Feet Following Brief Sun Exposure. J Clin Aesthet Dermatol. 2013;6(5):19-23.

4. Hardy JD, Solomon S., Banwell CS, Beach R, Wright V, Howard FM. Congenital complete heart block in the newborn associated with maternal systemic lupus erythematosus and other connective tissue disorders. Arch Dis Childhood. 1979;54(1):7-13.

5. Winkler RB, Nora AH, Nora JJ. Familial congenital complete heart block and maternal systemic lupus erythematosis. Circulation. 1977;56(6):1103-7.

6. McCredie M, Celermajer J, Sholler G, Kelly D, Chivers T, Wang Y, et al. A case-control study of congenital heart block: association with maternal antibodies to Ro(SS-A) and La(SS-B). Br J Rheumatol. 1990;29(1):10-4.

7. Brucato A. Prevention of congenital heart block in children of SSA-positive mothers. Rheumatology (Oxford). 2008;47(Suppl3):iii35-7.

8. Moutasim K, Shirlaw P, Escudier M, Poate T. Congenital heart block associated with Sjögren syndrome: case report. Int Arch Med. 2009;2(1):21.

9. Elish D, Silverberg NB. Neonatal lupus erythematosus. Cutis. 2006;77(2):82-6. 10. Boros CA, Spence D, Blaser S, Silverman ED. Hydrocephalus and macrocephaly: new manifestations of neonatal lupus erythematosus. Arthritis Rheum. 2007:57(2):261-6.

11. Izmirly PM, Saxena A, Kim MY, Wang D, Sahl SK, Llanos C, et al. Maternal and fetal factors associated with mortality and morbidity in a multi-racial/ ethnic registry of anti-SSA/Ro-associated cardiac neonatal lupus. Circulation. 2011;124(18):1927-35.

12. Morquio, L. Sur une maladie infantile et familiale characterisée par des modifications permanentes du pouls, des attaques syncopales et epileptiforme et la mort subite. Arch Méd d'Enfants. 1901;4:467.

13. Yater WM. Congenital heart block; review of the Literature; Report of a case with incomplete heterotaxy; the electrocardiogram in dextrocardia. Am J Dis Child 1929;38(1):112-36.

14. Mondal T, Dubey NK, Jajoo D, Dutta A, Sharma D. Congenital complete heart block. Indian Pediatr. 1995;32(11):1221-4.

15. Lazzerini PE, Capecchi PL, Guideri F, Acampa M, Galeazzi M, Laghi Pasini F. Connective tissue diseases and cardiac rhythm disorders: an overview. Autoimmun Rev. 2006;5(5):306-13.

16. Veille JC, Covitz W. Fetal cardiovascular hemodynamics in the presence of complete atrioventricular block. Am J Obstet Cynecol. 1994;170(5 Pt
1):1258-62.

17. Dipti TR, Azam MS, Sattar MH, Rahman SA. Detection of anti-nuclear antibody by immunofluorescence assay and enzyme immunoassay in childhood systemic lupus erythematosus: experience from Bangladesh. Int J Rheum Dis. 2012;15(1):121-5.

18. Gordon PA. Congenital heart block: clinical features and therapeutic approaches. Lupus. 2007;16(8):642-6.

19. Camm AJ, Bexton RS. Congenital complete heart block. Eur Heart J. 1984;5 (Suppl A):115-7

20. Moak JP, Barron KS, Hougen TJ, Wiles HB, Balajj S, Sreeram N, et al. Congenital heart: development of late-onset cardiomyopathy, a previously under appreciated sequela. J Am Coll Cardiol. 2001;37(1):238-242.

21. Edwards CS, Mootoo R, Bhanji A. High grade heart block in association with SLE. Ann Rheum Dis. 2004;63(5):606.

22. Chronister CS. Congenital complete atrioventricular block in a young man: a case study. Crit Care Nurse. 2009;29(5):45-56.

23. Ambrosi A, Wahren-Herlenius M. Congenital heart block: evidence for a pathogenic role of maternal autoantibodies. Arthritis Res Ther. 2012;14(2):208. 24. Jaeggi ET, Hamilton RM, Silverman ED, Zamora SA, Hornberger LK. Outcome of children with fetal, neonatal or childhood diagnosis of isolated congenital atrioventricular block. A single institution's experience of 30 years. J Am Coll Cardiol. 2002;39(1):130-7.

25. Buyon JP, Hiebert R, Copel J, Craft J, Friedman D, Katholi M, et al. Autoimmune-associated congenital heart block: demographics, mortality, morbidity and recurrence rates obtained from a national neonatal lupus registry. J Am Coll Cardiol. 1998;31(7):1658-66.

26. Guettrot-Imbert $\mathrm{G}$, Cohen L, Fermont L, Villain E, Frances C, Thiebaugeorges 0 , et al. A new presentation of neonatal lupus: 5 cases of isolated mild endocardial fibroelastosis associated with maternal Anti-SSA/Ro and Anti-SSB/La antibodies. J Rheumatol. 2011;38(2):378-86.

27. Breur JM, Visser GH, Kruize AA, Stoutenbeek P, Meijboom EJ. Treatment of fetal heart block with maternal steroid therapy: case report and review of the literature. Ultrasound Obstet Gynecol. 2004;24(4):467.

28. Epstein AE, DiMarco JP, Ellenbogen KA, Estes NA 3rd, Freedman RA, Gettes LS, et al. ACC/AHA/HRS 2008 Guidelines for Device-Based Therapy of Cardiac Rhythm Abnormalities: a report of the American College of Cardiology/American Heart Association Task Force on Practice Guidelines (Writing Committee to Revise the ACC/AHA/NASPE 2002 Guideline Update for Implantation of Cardiac Pacemakers and Antiarrhythmia Devices): developed in collaboration with the American Association for Thoracic Surgery and Society of Thoracic Surgeons. Circulation. 2008;117(21):e350-408.

29. Kaszala K, Huizar JF, Ellenbogen KA. Contemporary pacemakers: what the primary care physician needs to know. Mayo Clin Proc. 2008;83(10):1170-86.

\footnotetext{
Acknowledgments

The authors would like to thank RAK Medical at Health Sciences University, Saqr Hospital, Dr. S. Gurumadhva Rao Vice Chancellor of RAKHSU, Dr. Kannan Murugesan Dean of RAK College of Medical Sciences, Dr. Saleh Ahmed Ali Hassan of pediatric department at Saqr Hospital, Dr. Zita Lobo (English professor) RAKMHSU.
}

Conflict of Interest Statement t Funding

The Authors have no funding, financial relationships or conflicts of interest to disclose

Cite as:

Kiblawi M, Naeem A, Al Attrash E, Kar S, Goud B.K. Complete Congenital Heart Block in a Newborn associated with Maternal Systemic Lupus Erythematosus: A Case Report. Int J Med Students. 2013;1(3):128-31. 